ANL-HEP-CP-96-79

September 1996

\title{
LATTICE CALCULATION OF QUARKONIUM DECAY MATRIX ELEMENTS*
}

\author{
G.T. BODWIN AND D.K. SINCLAIR \\ HEP Division, Argonne National Laboratory, 9700 South Cass Avenue \\ Argonne, Illinois 60517, USA \\ S. KIM \\ Center for Theoretical Physics, Seoul National University \\ Seoul, Korea
}

\begin{abstract}
We calculate the NRQCD matrix elements for the decays of the lowest-lying S- and Pwave states of charmonium and bottomonium in quenched lattice QCD. We also compute the one-loop relations between the lattice and continuum matrix elements.
\end{abstract}

\section{Heavy-Quarkonium Formalism}

Heavy-quarkonium systems are nonrelativistic. In the CM frame, the average quark velocity $v$ satisfies $v^{2} \ll 1$, where $v^{2} \approx 0.3$ for charmonium, and $v^{2} \approx$ 0.1 for bottomonium. This fact allows one to describe heavy-quarkonium systems conveniently in terms of the effective field theory Nonrelativistic QCD (NRQCD) 1 NRQCD accurately describes processes in which $p_{Q}<M_{Q}$. Its utility stems from the fact that it can be used to decouple short-distance $\left(\sim 1 / M_{Q}\right)$ processes from long-distance $\left(\sim\right.$ quarkonium size $\left.\sim 1 /\left(M_{Q} v\right)\right)$ processes.

$Q \bar{Q}$ annihilation occurs at a distance of order $1 / M_{Q}$, so NRQCD does not describe the details of that process. In NRQCD, the short-distance part of the amplitude for $Q \bar{Q} \rightarrow$ light hadrons $\rightarrow Q \bar{Q}$ is pointlike, and the entire amplitude is described by a four-fermion interaction. The quarkonium total annihilation rate is proportional to the imaginary part of the matrix element in the quarkonium state of the appropriate four-fermion operator.

Coefficients of the four-fermion operators are determined by matching matrix elements in NRQCD to those in full QCD. The coefficients are short-distance quantities and, hence, are calculable in QCD perturbation theory. They are proportional to the IR-finite parts of the parton-level annihilation rates.

\subsection{Factorization theorems}

Using these ideas, Bodwin, Braaten, and Lepage

*Talk presented by G.T. Bodwin at the Quarkonium Physics Workshop, University of Illinois, Chicago, June 13-15, 1996. This work has been reported previously in Ref. 1. 
decay rate can be written as a sum of terms. Each term is the product of a longdistance matrix element of a four-fermion operator in the quarkonium state with a short-distance coefficient. For example, decay rates for S-wave quarkonia through next-to-leading order in $v^{2}$ are given by

$$
\begin{aligned}
\Gamma\left({ }^{2 s+1} S_{J} \rightarrow X\right) & =\mathcal{G}_{1}\left({ }^{2 s+1} S_{J}\right) 2 \operatorname{Im} f_{1}\left({ }^{2 s+1} S_{J}\right) / M_{Q}^{2} \\
& +\mathcal{F}_{1}\left({ }^{2 s+1} S_{J}\right) 2 \operatorname{Im} g_{1}\left({ }^{2 s+1} S_{J}\right) / M_{Q}^{4} .
\end{aligned}
$$

Decay rates for $\mathrm{P}$-wave quarkonia, to the lowest non-trivial order in $v^{2}$, are given by

$$
\begin{aligned}
\Gamma\left({ }^{2 s+1} P_{J} \rightarrow X\right) & =\mathcal{H}_{1}\left({ }^{2 s+1} P_{J}\right) 2 \operatorname{Im} f_{1}\left({ }^{2 s+1} P_{J}\right) / M_{Q}^{4} \\
& +\mathcal{H}_{8}\left({ }^{2 s+1} P_{J}\right) 2 \operatorname{Im} f_{8}\left({ }^{2 s+1} S_{J}\right) / M_{Q}^{2} .
\end{aligned}
$$

The $f$ 's and $g$ 's are the short-distance coefficients and are proportional to the rates for the annihilation of a $Q \bar{Q}$ pair from the ${ }^{2 s+1} L_{J}$ state. $\mathcal{G}_{1}, \mathcal{F}_{1}, \mathcal{H}_{1}$, and $\mathcal{H}_{8}$ are the long-distance matrix elements, which we calculate in this paper. The subscripts 1 and 8 indicate the color state of the $Q \bar{Q}$ pair. (An octet state, in lowest order in $v$, consists of a $Q \bar{Q} g$ state.)

\subsection{Matrix elements}

The long-distance matrix elements are defined by

$$
\begin{aligned}
& \mathcal{G}_{1}=\left\langle{ }^{1} S_{0}\left|\psi^{\dagger} \chi \chi^{\dagger} \psi\right|{ }^{1} S_{0}\right\rangle, \\
& \mathcal{F}_{1}=\left\langle{ }^{1} S_{0}\left|\psi^{\dagger} \chi \chi^{\dagger}\left(\frac{-i}{2} \stackrel{\leftrightarrow}{\mathbf{D}}\right)^{2} \psi\right|{ }^{1} S_{0}\right\rangle, \\
& \mathcal{H}_{1}=\left\langle{ }^{1} P_{1}\left|\psi^{\dagger}(i / 2) \stackrel{\leftrightarrow}{\mathbf{D}} \chi \cdot \chi^{\dagger}(i / 2) \stackrel{\leftrightarrow}{\mathbf{D}} \psi\right|{ }^{1} P_{1}\right\rangle, \\
& \mathcal{H}_{8}=\left\langle{ }^{1} P_{1}\left|\psi^{\dagger} T^{a} \chi \chi^{\dagger} T^{a} \psi\right|^{1} P_{1}\right\rangle .
\end{aligned}
$$

Here, $\psi$ and $\chi^{\dagger}$ are two-component spinors that annihilate a heavy quark and a heavy antiquark, respectively.

The $\mathcal{G}_{1}$ and $\mathcal{H}_{1}$ terms in the decay rates (11) and (2) appear in the conventional, color-singlet model. 6 For the color-singlet matrix elements we can, to good approximation, take only the vacuum in the intermediate state. 3 Then we have

$$
\begin{aligned}
& \mathcal{G}_{1}=\left|\left\langle{ }^{1} S_{0}\left|\psi^{\dagger} \chi\right| 0\right\rangle\right|^{2}\left(1+\mathcal{O}\left(v^{4}\right)\right), \\
& \mathcal{F}_{1}=\left\langle{ }^{1} S_{0}\left|\psi^{\dagger} \chi\right| 0\right\rangle\left\langle 0\left|\chi^{\dagger}\left(\frac{-i}{2} \stackrel{\leftrightarrow}{\mathbf{D}}\right)^{2} \psi\right|^{1} S_{0}\right\rangle\left(1+\mathcal{O}\left(v^{4}\right)\right), \\
& \mathcal{H}_{1}=\left|\left\langle{ }^{1} P_{1}\left|\psi^{\dagger} \frac{-i}{2} \stackrel{\leftrightarrow}{\mathbf{D}} \chi\right| 0\right\rangle\right|^{2}\left(1+\mathcal{O}\left(v^{4}\right)\right) .
\end{aligned}
$$

It then follows that, in the vacuum-saturation approximation,

$$
\begin{aligned}
\mathcal{G}_{1} & \approx \frac{3}{2 \pi}\left|R_{S}(0)\right|^{2}, \\
\mathcal{H}_{1} & \approx \frac{9}{2 \pi}\left|R_{P}^{\prime}(0)\right|^{2},
\end{aligned}
$$


where $R(0)$ is the radial wavefunction at the origin, and $R^{\prime}(0)$ is the derivative of the radial wavefunction at the origin. The matrix-elements (3) define a regularized $R(0)$ and a regularized $R^{\prime}(0)$ in QCD. The ratio $\mathcal{F}_{1} / \mathcal{G}_{1}$ measures the average of $\vec{p}^{2}$ in the quarkonium state.

The term in the $\mathrm{P}$-wave decay rate (2) that is proportional to $\mathcal{H}_{8}$ is absent in the color-singlet model. The matrix element $\mathcal{H}_{8}$ is proportional to the probability to find a $Q \bar{Q} g$ component in $\mathrm{P}$-wave quarkonium, with the $Q \bar{Q}$ pair in a relative $\mathrm{S}$-wave, color-octet state. It is perhaps the most interesting of the matrix elements that we measure, since it corresponds to a field-theoretic effect of QCD and is, therefore, inaccessible through any potential model of quarkonium.

\section{Lattice Measurement of the Matrix Elements}

\subsection{Euclidean-space formalism}

In Euclidean space, quarkonium two-point functions decay exponentially:

$$
\lim _{T \rightarrow \infty}\left\langle M(T) \tilde{M}^{\dagger}(0)\right\rangle=\lim _{T \rightarrow \infty}\left\langle 0\left|M e^{-H T} \tilde{M}^{\dagger}\right| 0\right\rangle=\langle 0|M| l\rangle e^{-E_{l} T}\left\langle l\left|\tilde{M}^{\dagger}\right| 0\right\rangle .
$$

Here, $M$ and $\tilde{M}$ are quarkonium sources, and $|l\rangle$ is the lowest-lying state with the quarkonium quantum numbers. We deduce from (6) that, in the vacuum-saturation approximation, the quarkonium matrix elements are proportional to the coefficients of $e^{-E_{l} T}$ in the appropriate two-point functions.

Similarly, a three-point function involving the four-fermion operator $\mathcal{O}$ has the behavior

$$
\begin{aligned}
\lim _{T, T^{\prime} \rightarrow \infty}\left\langle M\left(T+T^{\prime}\right) \mathcal{O}(T) M^{\dagger}(0)\right\rangle & =\langle 0|M| l\rangle e^{-E_{l} T^{\prime}}\langle l|\mathcal{O}| l\rangle e^{-E_{l} T}\left\langle l\left|M^{\dagger}\right| 0\right\rangle \\
& =\left\langle M\left(T+T^{\prime}\right) M^{\dagger}(0)\right\rangle\langle l|\mathcal{O}| l\rangle .
\end{aligned}
$$

Therefore, one can determine the expectation value of $\mathcal{O}$ in the quarkonium state by measuring the ratio of a three-point function to a two-point function.

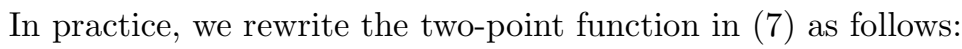

$$
\lim _{T, T^{\prime} \rightarrow \infty}\left\langle M\left(T+T^{\prime}\right) M^{\dagger}(0)\right\rangle=\frac{1}{C}\left\langle M\left(T+T^{\prime}\right) M_{\mathrm{p}}(T)\right\rangle\left\langle M_{\mathrm{p}}^{\dagger}(T) M^{\dagger}(0)\right\rangle,
$$

where $M_{P}$ is a point-source interpolating operator, and $C$ is the coefficient of $e^{-E_{l} T}$ in $\left\langle M \mathrm{p}(T) M_{\mathrm{p}}^{\dagger}(0)\right\rangle$. The sources in the three-point function match those in the twopoint functions on the right side of (8). Hence, we can reduce noise by measuring the ratio of the three-point function to those two-point functions. The expectation value $\langle l|\mathcal{O}| l\rangle$ is then represented by the diagram in Fig. 1.

\subsection{Computational method}

We measured operator expectation values using noisy-point and noisy-gaussian sources and generating retarded and advanced quark propagators from each time 


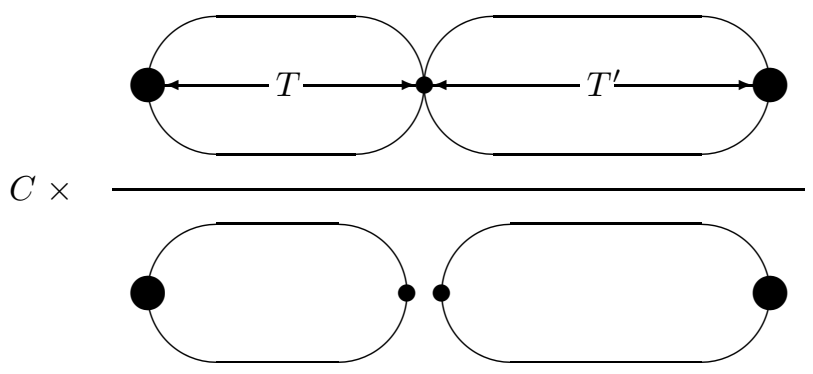

Fig. 1. Diagrammtic representation of $\langle l|\mathcal{O}| l\rangle$. The large disks represent quarkonium sources (and sinks); the small disks represent quarkonium point sources and four-fermion operators.

slice. We chose the Coulomb gauge for the field configurations. This gauge choice made the implementation of extended sources simpler, and it allowed us to replace covariant derivatives with normal derivatives, with errors of relative order $v^{2}$. For matrix elements of covariant operators, we checked some of our results on nongauge-fixed field configurations.

In calculating heavy-quark propagators $G(\mathbf{x}, t)$ on the lattice, we used the nonrelativistic formulation of Lepage, L. Magnea, Nakhleh, U. Magnea, Hornbostel We chose an evolution equation that is valid to the lowest non-trivial order in $v^{2}$, that is, a lattice version of the inhomogeneous Schrödinger equation:

$$
G(\mathbf{x}, t+1)=\left(1-H_{0} / 2 n\right)^{n} U_{\mathbf{x}, t}^{\dagger}\left(1-H_{0} / 2 n\right)^{n} G(\mathbf{x}, t)+\delta_{\mathbf{x}, \mathbf{0}} \delta_{t+1,0},
$$

with the initial condition $G(\mathbf{x}, t)=0$ for $t<0$. Here, $H_{0}=-\nabla^{(2)} /\left(2 M_{0}\right)-h_{0}$, $\nabla^{(2)}$ is the gauge-covariant discrete Laplacian, $M_{0}$ is the bare heavy-quark mass, $u_{0}=\left\langle(1 / 3) \operatorname{Tr} U_{\text {plaquette }}\right\rangle^{\frac{1}{4}}$ is the mean-field value of a gauge-field link, and $h_{0}=$ $3\left(1-u_{0}\right) / M_{0}$ is the mean-field energy shift. This chojce of $h_{0}$ is equivalent at leading order in $v$ to tadpole improvement of the action. 5 We chose $n=2$. For our choices of the bare masses $M_{0}$, this is the minimum value of $n$ for which the $Q \bar{Q}$ propagators are free from lattice-artifact singularities.

Note that $\mathcal{F}_{1} / M_{Q}^{2}$ is suppressed by $\mathcal{O}\left(v^{2}\right)$ relative to $\mathcal{G}_{1}$. That is, this ratio is of the same order as terms that we have neglected in the evolution equation. However, in ${ }^{3} S_{1} \rightarrow$ Light Hadrons, ${ }^{3} S_{1} \rightarrow \gamma+$ Light Hadrons, and ${ }^{3} S_{1} \rightarrow 3 \gamma$, the coefficient of $\mathcal{F}_{1} / M_{Q}^{2}$ is approximately -5 times that of $\mathcal{G}_{1}$. merit in calculating $\mathcal{F}_{1} / M_{Q}^{2}$, even in the presence of order $v^{2}$ errors.

\subsection{Parameters of the lattice simulation}

In our bottomonium and charmonium measurements, we used of 158 quenched gauge-field configurations on a $16^{3} \times 32$ lattice at $6 / g^{2}=5.7$. In the case of bottomonium, we also used 149 configurations on a $16^{3} \times 32$ lattice at $6 / g^{2}=6.0$. We took our values of the bare heavy-quark masses from the determination by the NRQCD collaboration: $M_{b 0}=1.5$ at $6 / g^{2}=6.0, M_{b 0}=2.7$ at $6 / g^{2}=5.7$, and $M_{c 0}=0.69$ 
at $6 / g^{2}=5.7$. (Note that our definition of $M_{0}$ is $u_{0}$ times that of the NRQCD collaboration.) We also used $u_{0}=0.87778701$ at $6 / g^{2}=6.0$ and $u_{0}=0.8608261760$ at $6 / g^{2}=5.7$.

In converting from lattice to physical units, we used the lattice spacings determined by the NRQCD collaboration. Their values are $a^{-1}=2.4 \mathrm{GeV}$ for bottomonium at $6 / g^{2}=6.0, a^{-1}=1.37 \mathrm{GeV}$ for bottomonium at $6 / g^{2}=5.7$, and $a^{-1}=1.23 \mathrm{GeV}$ for charmonium at $6 / g^{2}=5.7$. At a given coupling, the lattice spacings are different for charmonium and bottomonium because the quenched approximation leads to slight inconsistencies when one tries to use the physical spectra to fix the lattice spacing.

\subsection{Lattice results}

Our measurements revealed that the vacuum-saturation approximation is even more accurate than one would expect. For bottomonium at $6 / g^{2}=6.0$, corrections to the vacuum-saturation approximation for $\mathcal{G}_{1}$ are of relative size $1.3(1) \times 10^{-3}$. For charmonium at $6 / g^{2}=5.7$, the corrections are about $1 \%$. The corrections to the vacuum-saturation approximation for $\mathcal{H}_{1}$ are also small. We assumed that the vacuum-saturation approximation is accurate for $\mathcal{F}_{1}$ as well. The numerical results that we present for $\mathcal{G}_{1}, \mathcal{F}_{1}$, and $\mathcal{H}_{1}$ are the vacuum-saturation values.

The results of our lattice measurements of the matrix elements are shown in Table 1. (The subscript $L$ denotes lattice regularization.) The first error in each

Table 1. Lattice values of the NRQCD matrix elements.

\begin{tabular}{|l|c|c|c|}
\hline \hline & charmonium & \multicolumn{2}{|c|}{ bottomonium } \\
\hline $6 / g^{2}$ & 5.7 & 5.7 & 6.0 \\
\hline $\mathcal{G}_{1 L}$ & $0.1317(2)(12)$ & $0.9156(9)(65)$ & $0.1489(5)(12)$ \\
$\mathcal{F}_{1 L}($ non $) / \mathcal{G}_{1 L}$ & $1.2543(7)$ & $2.7456(8)$ & $1.3135(8)$ \\
$\mathcal{F}_{1 L}($ cov $) / \mathcal{G}_{1 L}$ & $0.5950(5)$ & $2.1547(7)$ & $0.8522(5)$ \\
$\mathcal{F}_{1 L}\left(\right.$ non $\left._{2}\right) / \mathcal{G}_{1 L}$ & $0.7534(4)$ & $1.2205(2)$ & $0.7775(5)$ \\
$\mathcal{F}_{1 L}\left(\right.$ cov $\left._{2}\right) / \mathcal{G}_{1 L}$ & $0.5201(3)$ & $1.1111(2)$ & $0.6659(3)$ \\
$\mathcal{H}_{1 L}$ & $0.0208(2)(20)$ & - & $0.0145(6)(20)$ \\
$\mathcal{H}_{8 L} / \mathcal{H}_{1 L}$ & $0.034(2)(8)$ & - & $0.0152(3)(20)$ \\
\hline \hline
\end{tabular}

quantity is statistical. Where two errors are given, the second error is an estimate of systematics associated with the parametrization of the functions used to fit to the propagators and with contamination from higher states. The arguments of $\mathcal{F}_{1 L}$ indicate different lattice representations of the operator. The argument cov denotes a tadpole-improved naive gauge-covariant operator; the argument non denotes the simple, gauge-noncovariant, finite-difference operator in the Coulomb gauge. The subscript 2 indicates a difference operator with spacings of two lattice units. This softer lattice Laplacian was useful in controlling regulator-artifact power divergences 
in the operator matrix element, which we shall discuss later.

\section{Relation of Lattice Matrix Elements to Continuum Matrix Elements}

At leading order in $v^{2}$, the lattice and continuum matrix elements of the operators that we measured are related as follows:

$$
\begin{aligned}
\mathcal{G}_{1 L} & =(1+\epsilon) \mathcal{G}_{1}, \\
\mathcal{F}_{1 L} & =(1+\gamma) \mathcal{F}_{1}+\phi \mathcal{G}_{1}, \\
\mathcal{H}_{1 L} & =(1+\iota) \mathcal{H}_{1}+\kappa \mathcal{H}_{8}, \\
\mathcal{H}_{8 L} & =(1+\eta) \mathcal{H}_{8}+\zeta \mathcal{H}_{1} .
\end{aligned}
$$

(The continuum-regulated matrix elements have no subscript.)

Note that, to leading order in $v^{2}, \mathcal{G}_{1 L}$ has no $\mathcal{F}_{1}$ component. In fact, if one were to try to compute the addmixture of $\mathcal{F}_{1}$, using the leading order NRQCD action that corresponds to (9), then the resulting inconsistencies in the treatment of $v^{2}$ corrections would lead to uncanceled IR divergences.

The coefficients in (10) relate different UV regularizations of the operators. Therefore, they are short-distance quantities and, hence, are perturbatively calculable. The coefficients $\epsilon, \gamma, \phi, \iota, \eta$ and $\zeta$ are of order $\alpha_{s}$, while $\kappa$ is of order $\alpha_{s}^{3}$.

We calculated these coefficients through order $\alpha_{s}$ (one loop) in tadpole-improved perturbation theory. $\mathrm{B}$ First, we obtained analytic expressions for the integrands. By carrying out the time components of the loop integrations analytically, we were able to identify the IR divergent pieces, which are identical in the lattice and continuum matrix elements. After subtracting these divergent pieces, we evaluated the remaining IR-finite integrals numerically using VEGAS.

Our numerical results (in lattice units) for the case of $\overline{M S}$ regularization of the continuum matrix elements are shown in Table 2. The accuracy of the coefficients of $\alpha_{s}$ is better than $1 \%$. The quantity $\zeta$ depends at one-loop order on the factorization scale, which we took to be $1.3 \mathrm{GeV}$ for charmonium and $4.3 \mathrm{GeV}$ for bottomonium. These values are approximately equal to the $\overline{M S}$ c-quark and b-quark masses, respectively.

Some of the coefficients of $\alpha_{s}$ in the expressions for $\phi$ appear to be large. However, in physical units, $\mathcal{G}_{1}$ has dimensions (mass) ${ }^{3}$ and $\mathcal{F}_{1}$ has dimensions (mass) ${ }^{5}$. Hence, we see from (10) that $\phi$ has dimensions of (mass) ${ }^{2}$. We can make $\phi$ dimensionless by dividing $\mathcal{F}_{1}$ and $\phi$ by $M_{Q}^{2}$. Similarly, we can make $\kappa$ and $\zeta$ dimensionless by dividing $\mathcal{H}_{1}$ and $\kappa$ by $M_{Q}^{2}$ and multiplying $\zeta$ by $M_{Q}^{2}$. Using the values, $M_{b}=5.0 \mathrm{GeV}$ and $M_{c}=1.5 \mathrm{GeV}$, we find that none of the dimensionless coefficients of $\alpha_{s}$ is large. We conclude that the perturbation series is reasonably behaved in one-loop order.

In setting the scale for $\alpha_{s}$, we made use of the method of Brodsky, Lepage and Mackenzie. 19 For coefficients that arise from a positive integrand, the scale is about $1 / a$. In the case of integrands without definite sign, large cancellations can 
Table 2. Coefficients relating lattice and continuum $(\overline{M S})$ matrix elements.

\begin{tabular}{|c|c|c|c|}
\hline & charmonium & \multicolumn{2}{|c|}{ bottomonium } \\
\hline $6 / g^{2}$ & 5.7 & 5.7 & 6.0 \\
\hline$\epsilon$ & $-0.7326 \alpha_{s}$ & $0.2983 \alpha_{s}$ & $-0.4877 \alpha_{s}$ \\
\hline$\gamma($ non $)$ & $-0.02578 \alpha_{s}$ & $-1.248 \alpha_{s}$ & $-0.9117 \alpha_{s}$ \\
\hline$\gamma(\operatorname{cov})$ & $-2.860 \alpha_{s}$ & $-2.192 \alpha_{s}$ & $-2.560 \alpha_{s}$ \\
\hline$\gamma\left(\right.$ non $\left._{2}\right)$ & $-0.2774 \alpha_{s}$ & $-1.096 \alpha_{s}$ & $-0.9236 \alpha_{s}$ \\
\hline$\phi($ non $)$ & $1.486 \alpha_{s}$ & $10.90 \alpha_{s}$ & $4.418 \alpha_{s}$ \\
\hline$\phi(\operatorname{cov})$ & $0.3928 \alpha_{s}$ & $9.808 \alpha_{s}$ & $3.325 \alpha_{s}$ \\
\hline$\phi\left(\right.$ non $\left._{2}\right)$ & $1.004 \alpha_{s}$ & $6.096 \alpha_{s}$ & $2.863 \alpha_{s}$ \\
\hline$\iota$ & $-0.7603 \alpha_{s}$ & $-1.852 \alpha_{s}$ & $-1.191 \alpha_{s}$ \\
\hline$\eta$ & $0.09157 \alpha_{s}$ & $-0.03728 \alpha_{s}$ & $0.06096 \alpha_{s}$ \\
\hline$\zeta$ & $-0.1785 \alpha_{s}$ & $-0.006011 \alpha_{s}$ & $-0.01862 \alpha_{s}$ \\
\hline
\end{tabular}

make the normalizing integral anomalously small and spoil the simplest scale-setting method. Therefore, we chose the scale to be $1 / a$ for all of the coefficients, taking $\alpha_{s}=\alpha_{V}(1 / a)=0.3552$ at $6 / g^{2}=5.7$ and $\alpha_{s}=\alpha_{V}(1 / a)=0.2467$ at $6 / g^{2}=6.0$.

\section{Continuum Matrix Elements}

Substituting the lattice matrix elements and the lattice-to-continuum coefficients into (10), we obtain the results for the continuum-regulated $(\overline{M S})$ matrix elements shown in the first two columns of Table 3. The first two errors are the statistical and systematic errors from the lattice measurements. The third error is the systematic error from the neglect of terms of higher order in $\alpha_{s}$ in the lattice-to-continuum coefficients.

Errors from the omission of terms of higher order in $v^{2}$ in the evolution equation and in the operator mixing have not been reported in the first two columns of Table 3. We expect these errors to be of order $10 \%$ for bottomonium and $30 \%$ for charmonium. In the case of $\mathcal{G}_{1 L}$, the NRQCD collaboration has given results that are accurate to next-to-leading order in $v^{2}$. The weighted averages of the singletand triplet-state values are $\mathcal{G}_{1 L}=0.133(4)$ for charmonium at $6 / g^{2}=5.7$ and $\mathcal{G}_{1 L}=0.144(4)$ for bottomonium at $6 / g^{2}=6.0$, which are in good agreement with our results. This suggests that the approximate effect of the corrections of higher order in $v^{2}$ is to split the values of the matrix elements for a multiplet of spin states, without changing their spin average.

The second column of Table 3 does not include errors that arise from uncertainties in the physical values of $a^{-1}$. We estimate, from the results of the NRQCD collaboration, that these errors are $7 \%$ for $\mathcal{G}_{1}$ in charmonium, $13 \%$ for $\mathcal{H}_{1}$ in charmonium, $13 \%$ for $\mathcal{G}_{1}$ in bottomonium, and $23 \%$ for $\mathcal{H}_{1}$ in bottomonium.

In addition, there are errors associated with the quenched approximation, for which we have no quantitative estimate. 
Table 3. Continuum-regulated $(\overline{M S})$ matrix elements.

\begin{tabular}{|c|c|c|c|}
\hline & \multicolumn{2}{|c|}{ lattice } & \multirow[t]{2}{*}{ experiment } \\
\hline & lattice units & physical units & \\
\hline \multicolumn{4}{|c|}{ charmonium $6 / g^{2}=5.7$} \\
\hline $\mathcal{G}_{1}$ & $0.1780(3)(16)\left({ }_{-259}^{+366}\right)$ & $0.3312(6)(30)\left({ }_{-483}^{+681}\right) \mathrm{GeV}^{3}$ & $0.36(3) \mathrm{GeV}^{3}$ \\
\hline $\mathcal{F}_{1} / \mathcal{G}_{1}$ & $0.05-0.54$ & $0.07-0.82 \mathrm{GeV}^{2}$ & $0.057 \mathrm{GeV}^{2}$ \\
\hline $\mathcal{H}_{1}$ & $0.0285(2)(27)\left({ }_{-42}^{+60}\right)$ & $0.0802(6)(77)\left({ }_{-118}^{+167}\right) \mathrm{GeV}^{5}$ & $0.077(19)(28) \mathrm{GeV}^{5}$ \\
\hline $\mathcal{H}_{8} / \mathcal{H}_{1}$ & $0.086(1)(6)\left({ }_{-32}^{+42}\right)$ & $0.057(1)(4)\left({ }_{-21}^{+27}\right) \mathrm{GeV}^{-2}$ & $0.095(31)(34) \mathrm{GeV}^{-2}$ \\
\hline \multicolumn{4}{|c|}{ bottomonium $6 / g^{2}=5.7$} \\
\hline $\mathcal{G}_{1}$ & $0.8279(8)(59)\left(\left(_{-848}^{+1066}\right)\right.$ & $2.129(2)(15)\left({ }_{-218}^{+274}\right) \mathrm{GeV}^{3}$ & $3.55(8) \mathrm{GeV}^{3}$ \\
\hline $\mathcal{F}_{1} / \mathcal{G}_{1}$ & $-3.7-0.2$ & $-6.9-0.4 \mathrm{GeV}^{2}$ & - \\
\hline \multicolumn{4}{|c|}{ bottomonium $6 / g^{2}=6.0$} \\
\hline $\mathcal{G}_{1}$ & $0.1692(6)(14)\left(\begin{array}{l}+126 \\
-110\end{array}\right)$ & $2.340(8)(19)\left(\begin{array}{l}+173 \\
-151\end{array}\right) \mathrm{GeV}^{3}$ & $3.55(8) \mathrm{GeV}^{3}$ \\
\hline $\mathcal{F}_{1} / \mathcal{G}_{1}$ & $-0.34-0.28$ & $-2.0-1.6 \mathrm{GeV}^{2}$ & - \\
\hline $\mathcal{H}_{1}$ & $0.0205(9)(28)\left({ }_{-19}^{+23}\right)$ & $1.63(7)(23)\left({ }_{-15}^{+19}\right) \mathrm{GeV}^{5}$ & - \\
\hline $\mathcal{H}_{8} / \mathcal{H}_{1}$ & $0.0151(2)(14)\left({ }_{-29}^{+33}\right)$ & $0.00262(3)(24)\left({ }_{-51}^{+57}\right) \mathrm{GeV}^{-2}$ & - \\
\hline
\end{tabular}

\subsection{Experimental values of the matrix elements}

The third column in Table 3 gives phenomenological results for the matrix elements. $\mathcal{G}_{1}$ was extracted from the measured decay rates for $J / \psi \rightarrow e^{+} e^{-}, \eta_{c} \rightarrow \gamma \gamma$ and $\Upsilon \rightarrow e^{+} e^{-}$(Ref. 10), using the expressions in given in Ref. 3. The value for $\mathcal{F}_{1} / \mathcal{G}_{1}$ for $J / \psi$ is from the calculation of Ko, Lee and Song. $11 \mathcal{H}_{1}$ and $\mathcal{H}_{8} / \mathcal{H}_{1}$ for $\chi_{c}$ are from Ref. 12. There is no published data for $\chi_{b}$ decays into light hadrons, photons, and/or leptons. To extract the phenomenological matrix elements for $\mathcal{G}_{1}$ and $\mathcal{H}_{1}$, we used the values $M_{b}$ (pole) $=5.0 \mathrm{GeV}$ (Ref. 7), $M_{c}$ (pole) $=1.5 \mathrm{GeV}$ (Ref. [13), $\alpha_{s}\left(M_{c}\right)=0.243, \alpha_{s}\left(M_{b}\right)=0.179, \alpha\left(M_{c}\right)=1 / 133.3$, and $\alpha\left(M_{b}\right)=1 / 132$.

In the third column of Table 3, the first error is experimental, and the second, where it is given, is theoretical. Where no theoretical error is given, it is at least as large as the uncertainty from the neglect of terms of higher order in $\alpha_{s}$ in the calculation of the short-distance coefficients. This uncertainty is of nominal size $25 \%$ for charmonium and $20 \%$ for bottomonium. Errors that arises from uncertainties in the heavy-quark masses have not been reported in the third column. The NRQCD collaboration quotes an error of $4 \%$ for the $b$-quark mass, which leads to errors of $8 \%$ in $\mathcal{G}_{1}$ and $16 \%$ in $\mathcal{H}_{1}$. In the case of the $c$-quark mass there is, as yet, no reliable determination from a lattice calculation.

\section{Discussion}

In the case of charmonium, our results for $\mathcal{G}_{1}, \mathcal{H}_{1}$, and $\mathcal{H}_{8} / \mathcal{H}_{1}$ are in agreement with experiment, but the errors are large. It is interesting to note that this agreement would have failed if we hadn't included the lattice-to-continuum corrections 
to the matrix elements.

The ratio $\mathcal{F}_{1} / \mathcal{G}_{1}$ is poorly determined, largely because of uncertainties in the coefficient $\phi$ that gives the mixing of $\mathcal{F}_{1}$ into $\mathcal{G}_{1}$. The mixing of $\mathcal{F}_{1}$ into $\mathcal{G}_{1}$ is power UV divergent. Since the mixing is UV dominated and begins at one loop, we expect it to be of order $\alpha_{s}$. On the other hand, in the continuum, $\mathcal{F}_{1} /\left(M^{2} \mathcal{G}_{1}\right)$ is of order $v^{2} \ll 1$. Therefore the effect of mixing on the ratio $\mathcal{F}_{1} /\left(M^{2} \mathcal{G}_{1}\right)$ is of relative order $\alpha_{s} / v^{2}$ and is large for both charmonium and bottomonium.

Nevertheless, we can conclude that $\mathcal{F}_{1} /\left(M^{2} \mathcal{G}_{1}\right)$ is no larger than $\mathcal{O}\left(v^{2}\right)$, in agreement with the NRQCD scaling rules. and negative for bottomonium. Note that, because the continuum matrix element $\mathcal{F}$ is gotten by subtracting UV divergences, it need not be positive.

For bottomonium, the lattice result for $\mathcal{G}_{1}$ is $35-40 \%$ below the experimental value. We know from the results of the NRQCD collaboration that at least part of this discrepancy is due to the quenched approximation. 4.4 There is good agreement between our results at $6 / g^{2}=5.7$ and $6 / g^{2}=6.0$, which confirms the expected renormalization-group scaling behavior.

Our results for the $\mathrm{P}$-wave matrix elements for bottomonium can be translated immediately into predictions for bottomonium decay rates 12 The values for individual matrix are probably subject to large corrections from the quenched approximation, but the ratio of octet to singlet matrix elements may be less susceptible to this source of error.

Aside from quenching, the largest uncertainties in the matrix elements come from neglect higher-order (in $\alpha_{s}$ ) corrections to the lattice-to-continuum coefficients. One might remedy this situation by using lattice methods to compute the relations between the lattice matrix elements and the momentum-subtracted continuum matrix elements nonperturbatively, as suggested by Martinelli and Sachrajda. . The $^{2}$ momentum-subtracted matrix elements could then be converted to $\overline{M S}$ matrix elements in continuum perturbation theory.

In the continuum, $\overline{M S}$ regularization of the operator matrix elements leads to renormalon ambiguities 15 These ambiguities are of the same order in $v^{2}$ as the matrix elements of operators of higher dimension. In the case of $H_{8}$, we expect such ambiguities to be small, since $H_{1}$ first mixes with $H_{8}$ in order $\alpha_{s}^{3}$. Renormalon ambiguities are absent in the case of hard-cutoff regulators, such as the lattice. That is, they are an artifact of the regulator (factorization) scheme that one chooses to define NRQCD. The consistency of NRQCD as an effective theory guarantees that regulator-scheme dependence is absent in physical quantities. Hence, renormalon ambiguities cancel in decay rates if one computes the NRQCD short-distance coefficients and the lattice-to-continuum coefficients to the same order in $\alpha_{s}$.

It is interesting that, for both charmonium and bottomonium, the values of $\mathcal{H}_{8} / \mathcal{H}_{1}$ that we obtain are in agreement with a crude phenomenology 3 In this phenomenology, one obtains $\mathcal{H}_{8}$ by solving the one-loop evolution equation for $\mathcal{H}_{8}$, under the assumption that $\mathcal{H}_{8}$ vanishes below a scale $M_{Q} v$. The one-loop evolution of the decay matrix element $\mathcal{H}_{8}$ is the same as for the corresponding production 
matrix element $\mathcal{H}_{8}^{\prime}$. This suggests that $\mathcal{H}_{8}^{\prime} \approx \mathcal{H}_{8}$. The production matrix element $\mathcal{H}_{8}^{\prime}$ can be extracted from CDF data for charmonium production 16 and from recent CLEO data.17 Using our value for $\mathcal{H}_{1}$, we obtain $\mathcal{H}_{8}^{\prime} / \mathcal{H}_{1}=0.042(19) \mathrm{GeV}^{-2}$ and $\mathcal{H}_{8}^{\prime} / \mathcal{H}_{1}=0.046(28) \mathrm{GeV}^{-2}$, respectively, both of which are in good agreement with our result for $\mathcal{H}_{8} / \mathcal{H}_{1}$.

\section{Acknowledgements}

We wish to thank G. Peter Lepage, John Sloan and Christine Davies for informative discussions and for access to some of their unpublished results. We also thank G. Peter Lepage for carrying out simulations to check our S-wave results. Our calculations were performed on the CRAY C-90 at NERSC, whose resources were made available to us through the Energy Research Division of the U. S. Department of Energy. This work was supported by the U.S. Department of Energy, Division of High Energy Physics, Contract W-31-109-ENG-38. S. K. is supported by KOSEF through CTP.

\section{References}

1. G.T. Bodwin, S. Kim and D.K. Sinclair, in Lattice '94, Proceedings of the International Symposium, Bielefeld, Germany, edited by F. Karsch et al. [Nucl. Phys. B (Proc. Suppl.) 42 (1995) 306], Report No. hep-lat/9412011; Phys. Rev. Lett. 77 (1996) 2376.

2. W.E. Caswell and G.P. Lepage, Phys. Lett. 167B (1986) 437.

3. G.T. Bodwin, E. Braaten and G.P. Lepage, Phys. Rev. D 51 (1995) 1125.

4. For a review of the color-singlet model see G.A. Schuler, CERN Report No. CERNTH.7170/94 (hep-ph/9403387).

5. G.P. Lepage, et al., Phys. Rev. D 46 (1992) 4502.

6. G.T. Bodwin, E. Braaten and G.P. Lepage (unpublished).

7. C.T.H. Davies et al., Phys. Rev. D 50 (1994) 6963; Phys. Rev. Lett. 73 (1994) 2654; Phys. Rev. D 52 (1995) 6519; in Lattice '95, Proceedings of the International Symposium, Melbourne, Australia, edited by T.D. Kieu et al. [Nucl. Phys. B (Proc. Suppl.) 47 (1996) 409], Report No. hep-lat/9510006; ibid. 47 (1996) 421, Report No. heplat/9510052.

8. G.P. Lepage and P.B. Mackenzie, Phys. Rev. D 48 (1993) 2250.

9. S.J. Brodksy, G.P. Lepage, and P.B. Mackenzie, Phys. Rev. D 28, (1983) 228.

10. Review of Particle Properties, Phys. Rev. D 50 (1994) 1173.

11. P. Ko, J. Lee, and H.S. Song, Phys. Rev. D 53 (1996) 1409.

12. G.T. Bodwin, E. Braaten and G.P. Lepage, Phys. Rev. D 46 (1992) R1914.

13. E. J. Eichten and C. Quigg, Phys. Rev. D 52 (1995) 1726.

14. G.P. Lepage, private communication.

15. C.T. Sachrajda, in Lattice '95 (Ref. đ), p. 100, Report No. hep-lat/9509085, and references therein.

16. P. Cho and A.K. Leibovich, Phys. Rev. D 53 (1996) 6203.

17. R. Balest et al., Phys. Rev. D 52 (1995) 2661. 